\title{
Die Geschichte der SGAM oder Das Ende der Sprachlosigkeit in der Hausarztmedizin
}

\author{
Die Schweizerische Gesellschaft für Allgemeinmedizin (SGAM) hat sich vor \\ allem in den letzten Jahren stark als Sprachrohr der Schweizer Hausärzte pro- \\ filiert, indem sie die Hausarztmedizin zum nationalen Politikum machte und \\ der Hausärztebewegung neuen Schub verlieh. Dennoch ist der Hausärzte- \\ mangel nicht vom Tisch.
}

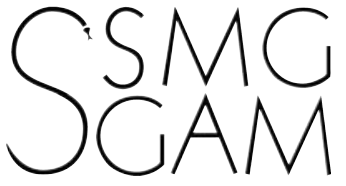

Bernhard Stricker, Medienbeauftragter SGAM
«Bundesrat schwächt Hausarztmedizin!» Unter diesem Titel verschickte die SGAM am 9. November 2005 ein Communiqué an die Schweizer Medien, worin sie gegen den gleichentags vom Bundesrat gefällten Entscheid protestierte, den Taxpunktwert für Laboruntersuchungen in Hausarztpraxen um 10\% zu senken, und kündigte an: «Die SGAM wird sich im Interesse der Patienten und einer qualitativ hochwertigen Hausarztmedizin diesem Beschluss widersetzen.»

Rund drei Wochen später, am 5. Dezember 2005, veröffentlichte die SGAM ein weiteres Communiqué, worin sie Kampfmassnahmen ankündigte: «SGAM ruft im Namen aller Hausärztinnen und Hausärzte der Schweiz zum Widerstand gegen die Schwächung der Hausarztmedizin und den drohenden Hausärztemangel auf.» Sie lancierte dazu eine Petition und begann, eine nationale Grosskundgebung am 1. April 2006 vor dem Bundeshaus in Bern vorzubereiten. Dazu holte sie die SGIM, das KHM, die SGP und die FMP ins Boot.

\section{Hohes Risiko}

Damit ging der SGAM-Vorstand ein hohes Risiko ein, denn die Auswirkungen einer solchen Aktion waren Anfang Dezember 2005 noch nicht absehbar: Was, wenn die Demo ein Flop wird? Die Glaubwürdigkeit stand auf dem Spiel. Doch der Leidensdruck war grösser als die Angst vor dem Versagen: Jahrelang angestaute Wut über die schleichende Demontage eines ganzen Berufsstandes durch Versicherer und Bundesbehörden brachten das Fass - «dank» des CouchepinDekrets - zum Überlaufen.

Der Rest ist inzwischen öffentlich bekannt: Der 1. April 2006 übertraf alle Erwartungen: 12000 Teilnehmer an der Kundgebung, über 300000 Unterschriften für die Petition. Fortan sollte es jedes Jahr am 1. April einen «Tag der Hausarztmedizin» geben.
}

\section{Jahre SGAM}

Die Gründung der SGAM geht auf einem Beschluss der Ärztekammer vom 27. Juni 1965 zurück, an der die FMH den Facharzttitel «Arzt für Allgemeinmedizin FMH» einführte und parallel dazu das erste Weiterbildungsprogramm verabschiedete, das am 1. Januar 1966 in Kraft gesetzt wurde.

Es dauerte weitere zwölf Jahre, bis sich die SGAM am 26. Oktober 1978 an ihrer ersten Generalversammlung in Luzern konstituierte.

\section{Gesundheitspolitische Positionen}

In ihren am 1. April 2008 verbreiteten «Gesundheitspolitischen Positionen» fordert die SGAM eine neue nationale Gesundheitspolitik, «in der die Hausärzte auch politisch jene zentrale Rolle übernehmen, die sie in der medizinischen Grundversorgung bereits heute haben». Ferner hält sie fest: «Die zunehmende Machtkonzentration der Versicherer, deren Jagd nach guten Risiken, verbunden mit hemmungsloser Gewinnmaximierung, bedrohen nicht nur den Grundgedanken des KVG, sondern ebenso sehr die medizinische Grundversorgung.»

\section{Ein Beruf, ein Ziel, eine Stimme}

An der SGAM-GV 2007 in St. Gallen beschlossen die Delegierten, die bisherige SGAM in eine neue Häusärztorganisation «Hausärzte Schweiz» überzuführen, in der Allgemeinmediziner, Internisten und Pädiater zusammengeschlossen sind, so wie das schon in zahlreichen Kantonen der Fall ist. Motto: ein Beruf, ein Ziel, eine Stimme.

Je eher und besser es den Schweizer Hausärzten auch auf nationaler Ebene gelingt, ihre Interessen in einem Verband zu bündeln, desto schneller erreichen sie ihr Ziel, die Hausarztmedizin deutlich aufzuwerten und die ihr gebührende Bedeutung auf Dauer aufrechtzuerhalten. 